\title{
Modified Hybrid Freeman/Eigenvalue Decomposition for Polarimetric SAR Data
}

\author{
Shuang Zhang, Shuang Wang, and Bo Chen \\ Key Laboratory of Intelligent Perception and Image Understanding of Ministry of Education, \\ International Research Center for Intelligent Perception and Computation, Xidian University, Xian, Shaanxi 710071, China \\ Correspondence should be addressed to Shuang Zhang; shzhang_work@163.com
}

Received 25 November 2014; Accepted 30 November 2014

Academic Editor: Gwanggil Jeon

Copyright (C) 2015 Shuang Zhang et al. This is an open access article distributed under the Creative Commons Attribution License, which permits unrestricted use, distribution, and reproduction in any medium, provided the original work is properly cited.

\begin{abstract}
Because of the rapid advancement of the airborne sensors and spaceborne sensors, large volumes of fully polarimetric synthetic aperture radar (PolSAR) data are available, but they are too complex to interpret difficultly. In this paper, a modified hybrid Freeman/eigenvalue decomposition method for the coherency matrix derived from the fully PolSAR sensors is proposed. The proposed modified hybrid Freeman/eigenvalue decomposition uses a real unitary transformation on the coherency matrix to release correlations between the copolarized term and cross polarized term, and the scattering models are derived from eigenvectors of the coherency matrix with reflection symmetry condition. The anisotropy and entropy are used to determine whether the volume scattering component is derived from the man-made structures or not. Moreover, the scattering powers from the proposed hybrid Freeman/eigenvalue decomposition are all nonnegative values. Fully PolSAR data on San Francisco acquired by AIRSAR sensor are used in the experiments to prove the efficacy of the proposed decomposition.
\end{abstract}

\section{Introduction}

Since 1985, the first fully polarimetric AIRSAR at L-band was launched by the Jet Propulsion Laboratory (JPL) [1] which started a rapid advancement stage of PolSAR sensors, such as the well-known airborne sensors: EMISAR [2] by the Technical University of Denmark and E-SAR [3] by DLR of Germany, and the famous spaceborne sensors: TerraSAR-X [4] by DLR of Germany and Radarsat-2 [5] by CSA of Canada and so forth. Large volumes of the fully PolSAR data need to be interpreted. Polarimetric target decomposition is an important and useful tool for understanding the PolSAR data [6] by separating received measurements into basic scattering mechanisms. The scattering mechanisms of the PolSAR media are analyzed for the purpose of parameter inversion, terrain classification, and so forth. Currently, eigenvector-based decompositions and model-based decomposition methods are commonly used on the second-order statistics matrix of the PolSAR data. Cloude and Pottier developed the most notable eigenvector-based decomposition, that is, Entropy/Alpha method [7]. The classical model-based decomposition was Freeman-Durden decomposition (FDD) developed by Freeman and Durden [8], which decomposed the coherency matrix of PolSAR data into three components: surface scattering, double-bounce scattering, and volume scattering with the reflection symmetry condition. The reflection symmetry condition implies that the correlation between copolarized term and cross polarized term is zero. FreemanDurden decomposition is used in various applications since it is easy to understand and accomplish.

When applying Freeman-Durden decomposition on the real PolSAR data, some scattering powers are negative those most frequently occur in the double-bounce scattering powers. To solve this deficiency, various modified methods have been developed [9-15]. Cloude improved Freeman-Durden decomposition via setting surface scattering model and double-bounce scattering model to be orthogonal [14], that is, well-known hybrid Freeman/eigenvalue decomposition. After the rotation of the coherency matrix, the scattering powers derived from hybrid Freeman/eigenvalue decomposition are effective at avoiding negative values. Singh improved the hybrid Freeman/eigenvalue decomposition by using different volume scattering models [15] for vegetation areas and oriented structures. 
In this paper, a modified version of hybrid Freeman/ eigenvalue decomposition for PolSAR data is proposed by using different volume scattering models for the vegetation areas and man-made structures. Moreover, the surface scattering model and double-bounce scattering model are defined as the eigenvectors of the coherency matrix of PolSAR data. The eigenvector with scattering angle $\alpha$ greater than $\pi / 4$ denotes the double-bounce scattering model, while the other scattering angle $\alpha$ less than $\pi / 4$ represents the surface scattering model. The volume scattering model for the manmade structures is also derived from the eigenvectors of the coherency matrix. We will show how the eigenspace of the coherency matrix enables the proposed Freeman/eigenvalue decomposition and solve the scattering powers as the linear combinations of eigenvalues. In addition, the scattering powers are all nonnegative values.

The rest of this paper is organized as follows. The hybrid Freeman/eigenvalue decomposition is summarized in Section 2. The proposed decomposition is presented in Section 3. Results and discussion of experiments performed on the real PolSAR data are provided in Section 4. Section 5 presents our conclusions.

\section{Freeman/Eigenvalue Decomposition}

In this section, the Freeman/eigenvalue decomposition is simply introduced for the integrity of this paper. The detail content has been shown in [14].

For monostatic PolSAR sensor with $\{H, V\}$ basis, a Pauli vector is used to represent the single look PolSAR data as (1) and the coherency matrix is used to represent the multilook PolSAR data as (2) as follows:

$$
\begin{gathered}
\vec{k}_{p}=\frac{1}{\sqrt{2}}\left[S_{H H}+S_{V V} S_{H H}-S_{V V} 2 S_{H V}\right]^{t} \\
\langle[T]\rangle=\left\langle\vec{k}_{p} \cdot \vec{k}_{p}^{* t}\right\rangle=\left[\begin{array}{lll}
T_{11} & T_{12} & T_{13} \\
T_{12}^{*} & T_{22} & T_{23} \\
T_{13}^{*} & T_{23}^{*} & T_{33}
\end{array}\right],
\end{gathered}
$$

where $t$ denotes a transposition operator, $*$ implies a complex conjugation processing, and \langle\rangle denotes the multilook processing.

In the model-based decomposition, such as FreemanDurden decomposition [8] and hybrid Freeman/eigenvalue decomposition [14], the measured coherency matrix of the PolSAR data is expanded into three components, surface scattering, double-bounce scattering, and volume scattering as follows:

$$
\langle[T]\rangle=m_{s} T_{s}+m_{d} T_{d}+m_{v} T_{v},
$$

where $T_{s}, T_{d}$, and $T_{v}$ are surface scattering model, doublebounce scattering model, and volume scattering model, respectively.

In hybrid Freeman/eigenvalue decomposition, those three scattering models are defined in (4), (5), and (6). $m_{s}, m_{d}$, and $m_{v}$ are the corresponding scattering powers in the proper order:

$$
\begin{aligned}
& T_{s} \\
& =\left[\begin{array}{ccc}
\cos ^{2} \alpha_{s} & \cos \alpha_{s} \sin \alpha_{s} e^{-j \phi_{s}} & 0 \\
\cos \alpha_{s} \sin \alpha_{s} e^{j \phi_{s}} & \sin ^{2} \alpha_{s} & 0 \\
0 & 0 & 0
\end{array}\right], \quad \alpha_{s} \leq \frac{\pi}{4}, \\
& T_{d} \\
& =\left[\begin{array}{ccc}
\cos ^{2} \alpha_{d} & \cos \alpha_{d} \sin \alpha_{d} e^{-j \phi_{d}} & 0 \\
\cos \alpha_{d} \sin \alpha_{d} e^{j \phi_{d}} & \sin ^{2} \alpha_{d} & 0 \\
0 & 0 & 0
\end{array}\right], \quad \alpha_{d}>\frac{\pi}{4}, \\
& T_{v}=\left[\begin{array}{ccc}
F_{s} & 0 & 0 \\
0 & 1 & 0 \\
0 & 0 & 1
\end{array}\right]
\end{aligned}
$$

$\alpha_{s}$ and $\alpha_{d}$ imply the type of the scattering model; namely, $\alpha_{s} \leq \pi / 4$ denotes the surface scattering, while $\alpha_{d}>$ $\pi / 4$ represents the double-bounce scattering. In [14], the condition $\alpha_{s}+\alpha_{d}=\pi / 2$ is set to reduce the number of unknowns. $F_{s}$ is the volume parameter. If $F_{s}=2$, hybrid Freeman/eigenvalue decomposition becomes equivalent to Freeman-Durden decomposition [8]. The cross polarized term $T_{33}$ only exists in the volume scattering model, so the volume scattering power $m_{d}$ is solved as follows:

$$
m_{v}=T_{33} .
$$

Since the rank of either surface scattering model $T_{s}$ or double-bounce scattering model $T_{d}$ is equal to 1 , the corresponding scattering powers $m_{s}$ and $m_{d}$ are the eigenvalues of $T_{\mathrm{SD}}$ as (10), and $m_{s}$ and $m_{d}$ can be solved as (11).

$\alpha_{s}$ and $\alpha_{d}$ are obtained as follows:

$$
\alpha_{d, s}=\cos ^{-1}\left[\left(1+\left|\frac{T_{12}}{T_{22}-T_{33}-m_{d, s}}\right|^{2}\right)^{-1 / 2}\right] .
$$

Whichever of $m_{s}$ and $m_{d}$ is smaller is always set to zero, and then volume parameter $F_{s}$ can be estimated as

$$
F_{s}=\frac{T_{11} \cdot\left(T_{22}-T_{33}\right)-\left|T_{12}\right|^{2}}{T_{33} \cdot\left(T_{22}-T_{33}\right)} .
$$

It enables solving for the scattering powers $m_{s}$ and $m_{d}$ by substituting (9) into (11):

$$
\begin{aligned}
T_{\mathrm{SD}}= & \langle[T]\rangle-m_{v} T_{v} \\
= & {\left[\begin{array}{ccc}
\cos \alpha & \sin \alpha & 0 \\
-\sin \alpha & \cos \alpha & 0 \\
0 & 0 & 1
\end{array}\right] \cdot\left[\begin{array}{ccc}
m_{s} & 0 & 0 \\
0 & m_{d} & 0 \\
0 & 0 & 0
\end{array}\right] } \\
& \cdot\left[\begin{array}{ccc}
\cos \alpha & -\sin \alpha & 0 \\
\sin \alpha & \cos \alpha & 0 \\
0 & 0 & 1
\end{array}\right]
\end{aligned}
$$




$$
\begin{aligned}
& m_{d, s} \\
& =\left(\left(T_{11}+T_{22}-\left(F_{s}+1\right) T_{33}\right)\right. \\
& \left.\quad \pm \sqrt{\left(T_{11}-T_{22}-\left(F_{s}-1\right) T_{33}\right)^{2}+4\left|T_{12}\right|^{2}}\right) \\
& \cdot(2)^{-1} \\
& \langle[T(\theta)]\rangle \\
& =\left[\begin{array}{ccc}
1 & 0 & 0 \\
0 & \cos 2 \theta & \sin 2 \theta \\
0 & -\sin 2 \theta & \cos 2 \theta
\end{array}\right]\langle[T]\rangle\left[\begin{array}{ccc}
1 & 0 & 0 \\
0 & \cos 2 \theta & -\sin 2 \theta \\
0 & \sin 2 \theta & \cos 2 \theta
\end{array}\right] .
\end{aligned}
$$

\section{Proposed Decomposition}

3.1. Rotation of the Coherency Matrix. To reduce the power of cross polarized term $T_{33}$, a real unitary transformation has been proposed to be implemented on the coherency matrix $[12,16]$ before decomposition as (12).

$\theta$ is the angle of rotation about the radar line of sight. To minimize $T_{33}$, two angles are solved as

$$
\theta=\frac{1}{4} \tan ^{-1}\left(\frac{2\left(\operatorname{Re}\left(T_{23}\right)\right)}{T_{22}-T_{33}}\right)
$$

After the rotation of the coherency matrix, the imagery element of $T_{23}$ is equal to zero.

3.2. Eigenvalue Decomposition of the Rotated Coherency Matrix. We assume that the reflection symmetry condition (i.e., $\left\langle S_{H H} S_{H V}^{*}\right\rangle \approx\left\langle S_{V V} S_{H V}^{*}\right\rangle \approx 0$ ) holds; then each pixel for multilook PolSAR data is represented as

$$
\langle[T(\theta)]\rangle=\left[\begin{array}{ccc}
T_{11}(\theta) & T_{12}(\theta) & 0 \\
T_{12}^{*}(\theta) & T_{22}(\theta) & 0 \\
0 & 0 & T_{33}(\theta)
\end{array}\right] .
$$

The average coherency matrix after rotation is decomposed [7] as

$$
\langle[T(\theta)]\rangle=U \cdot\left[\begin{array}{ccc}
\lambda_{1} & 0 & 0 \\
0 & \lambda_{2} & 0 \\
0 & 0 & \lambda_{3}
\end{array}\right] \cdot U^{* t}
$$

where $\lambda_{1}, \lambda_{2}$, and $\lambda_{3}$ are the eigenvalues of the rotated coherency matrix $\langle[T(\theta)]\rangle$, and $\lambda_{1} \geq \lambda_{2} \geq \lambda_{3} \geq 0$. Because of the reflection symmetry condition and the rotation of the coherency matrix, $U$ consists of the corresponding eigenvectors $k_{1}, k_{2}$, and $k_{3}$ as

$$
U=\left[\begin{array}{lll}
k_{1} & k_{2} & k_{3}
\end{array}\right]=\left[\begin{array}{ccc}
\cos \alpha_{1} & \cos \alpha_{2} & 0 \\
\sin \alpha_{1} e^{j \gamma_{1}} & \sin \alpha_{2} e^{j \gamma_{2}} & 0 \\
0 & 0 & 1
\end{array}\right],
$$

where eigenvectors $k_{1}, k_{2}$, and $k_{3}$ are orthogonal unit vectors, $\alpha_{1}+\alpha_{2}=\pi / 2$.
3.3. Scattering Models. Because in hybrid Freeman/eigenvalue decomposition, the condition $\alpha_{s}+\alpha_{d}=\pi / 2$ is set, and, in eigenvectors of the coherency matrix, it can be seen that $\alpha_{1}+\alpha_{2}=\pi / 2$, we can draw the conclusions: the eigenvectors $k_{1}$ and $k_{2}$ contain a surface scattering component and a double-bounce scattering component, and the scattering angles $\alpha_{1}$ and $\alpha_{2}$ are equivalent to the angles $\alpha_{s}$ and $\alpha_{d}$ of the hybrid Freeman/eigenvalue decomposition. The eigenvector $k_{1}$ or $k_{2}$ in $U$ with $\alpha \leq \pi / 4$ is shown in (17) which denotes the surface scattering targets, and the other eigenvector with $\pi / 4 \leq \alpha \leq \pi / 2$ is a double-bounce scattering target (18) under the reflection symmetry condition:

$$
\begin{aligned}
& k_{s}=\left[\begin{array}{c}
\cos \alpha_{s} \\
\sin \alpha_{s} e^{j \gamma_{s}} \\
0
\end{array}\right], \quad \alpha_{s} \leq \frac{\pi}{4}, \\
& k_{d}=\left[\begin{array}{c}
\cos \alpha_{d} \\
\sin \alpha_{d} e^{j \gamma_{d}} \\
0
\end{array}\right], \quad \frac{\pi}{4} \leq \alpha_{d} \leq \frac{\pi}{2} .
\end{aligned}
$$

If $\alpha_{1} \leq \pi / 4$, we obtain $\alpha_{s}=\alpha_{1}$, the corresponding eigenvector $k_{s}=k_{1}$ as (17), and surface scattering model $T_{s}=k_{1} k_{1}^{* t}$ whose form is the same as the one in (4); $\alpha_{d}=$ $\pi / 2-\alpha_{1}=\alpha_{2}$ and double-bounce scattering model $T_{d}=k_{2} k_{2}^{* t}$ is identical to (5). In other cases, if $\alpha_{2} \leq \pi / 4, \alpha_{s}=\alpha_{2}$, surface scattering model $T_{s}=k_{2} k_{2}^{* t}$ as (4), $\alpha_{d}=\pi / 2-\alpha_{2}=\alpha_{1}$, and double-bounce scattering $T_{d}=k_{1} k_{1}^{* t}$ as (5).

3.3.1. Volume Scattering Model from the Man-Made Structures. The entropy and anisotropy are defined in the eigenspace of the coherency matrix to measure the randomness of the targets [7, 17]:

$$
\begin{gathered}
H=-\sum_{I=1,2,3} p_{i} \log 3\left(p_{i}\right), \\
\text { with } p_{i}=\frac{\lambda_{i}}{\left(\lambda_{1}+\lambda_{2}+\lambda_{3}\right)}, \\
A=\frac{\left(\lambda_{2}-\lambda_{3}\right)}{\left(\lambda_{2}+\lambda_{3}\right)} .
\end{gathered}
$$

For the man-made structures, usually the value of entropy $(H)$ is large (usually $H>0.7$ ), and anisotropy is often larger than $0.5[7,17]$. Based on these, we defined the volume scattering model as

$$
T=\frac{1}{2}\left[\begin{array}{lll}
1 & 0 & 0 \\
0 & 0 & 0 \\
0 & 0 & 1
\end{array}\right]
$$

The rank of the volume scattering model is equal to two. The volume scattering model is designed as a distributed target that is diffused from the surface scatterers whose scattering angle $\alpha=0$ and orientation angle $\beta=0$ and the oriented objects whose scattering angle $\alpha=90$ and orientation angle $\beta=90$. For the real PolSAR data, the oriented objects can be modeled as angle $\alpha=90$ and orientation angle $\beta=90$ because of the reflection symmetry 
condition. But the surface scatterers usually are not the same as " $\alpha=0$ and $\beta=0$," so the surface scattering case is relaxed as (17), and the volume scattering model for the man-made structures is defined as

$$
T_{v_{-} 2}=\frac{1}{2}\left(k_{s} k_{s}^{* t}+k_{3} k_{3}^{* t}\right)
$$

3.3.2. Volume Scattering Model from the Vegetation Areas. The volume scattering model is used as a unit diagonal matrix whose rank is equal to 3 which has been proposed by An et al. [12]. Because the unit diagonal matrix has the largest entropy $(H=1)$ and least anisotropy $(A=0)$, the unit diagonal matrix is also used for the vegetation areas in this paper:

$$
T_{v_{-} 1}=\frac{1}{3}\left[\begin{array}{lll}
1 & 0 & 0 \\
0 & 1 & 0 \\
0 & 0 & 1
\end{array}\right] \text {. }
$$

3.4. Freeman/Eigenvalue Decomposition of the Rotated Coherency Matrix. For vegetation areas, the cross polarized term only presents in the volume scattering model, and volume scattering power can be solved as $m_{v}=3 T_{33}(\theta)$. In the eigenspace of the rotated coherency matrix under the reflection symmetry condition, the cross polarized term $T_{33}(\theta)$ is one of the eigenvalues. After the real unitary transformation, $T_{33}(\theta)$ reaches the minimum value, usually, $T_{33}(\theta)=\lambda_{3}$. So the volume scattering power is solved as

$$
m_{v}=3 \lambda_{3}
$$

The surface scattering power $m_{s}$ and double-bounce scattering power $m_{d}$ are the eigenvalues of $T_{\mathrm{SD}}$. According to the eigenvalue decomposition of the rotated coherency matrix with reflection symmetry condition, $T_{\mathrm{SD}}$ can be expanded as

$$
\begin{aligned}
\langle[T(\theta)]\rangle_{\mathrm{SD}} & =m_{s} T_{s}+m_{d} T_{d} \\
& =\langle[T(\theta)]\rangle-m_{v} T_{v_{-} 1} \\
& =\langle[T(\theta)]\rangle-3 \lambda_{3} T_{v_{-} 1} \\
& =\left(\lambda_{1}-\lambda_{3}\right) k_{1} k_{1}^{* t}+\left(\lambda_{2}-\lambda_{3}\right) k_{2} k_{2}^{* t} .
\end{aligned}
$$

From (23), $\alpha_{1} \leq \pi / 4$, the surface scattering power $m_{s}$, and double-bounce scattering power $m_{d}$ are solved as (25) while $\alpha_{2} \leq \pi / 4, m_{s}$, and $m_{d}$ are solved as (26):

$$
\begin{aligned}
& m_{s}=\lambda_{1}-\lambda_{3}, \\
& m_{d}=\lambda_{2}-\lambda_{3}, \\
& m_{s}=\lambda_{2}-\lambda_{3}, \\
& m_{d}=\lambda_{1}-\lambda_{3} .
\end{aligned}
$$

For man-made structures, that is, $H<0.7$ and $A>0.5$ $[7,17]$, similar to the case of vegetation areas, the volume scattering power is solved as

$$
m_{v}=2 \lambda_{3}
$$

Expand the coherency matrix after the real unitary transformation into eigenspace:

$$
\begin{aligned}
\langle[T(\theta)]\rangle= & m_{s} T_{s}+m_{d} T_{d}+m_{v} T_{v} \\
= & \langle[T(\theta)]\rangle_{\mathrm{SD}}+m_{v} T_{v_{-} 1} \\
= & \langle[T(\theta)]\rangle_{\mathrm{SD}}+2 \cdot \lambda_{3} \cdot \frac{1}{2}\left(k_{s} k_{s}^{* t}+k_{3} k_{3}^{* t}\right) \\
= & \lambda_{1} k_{1} k_{1}^{* t}+\lambda_{2} k_{2} k_{2}^{* t}+\lambda_{3} k_{3} k_{3}^{* t} \\
= & \left(\lambda_{1}-\lambda_{2}\right) k_{1} k_{1}^{* t}+\lambda_{2} k_{2} k_{2}^{* t} \\
& +2 \cdot \lambda_{3} \cdot \frac{1}{2}\left(k_{1} k_{1}^{* t}+k_{3} k_{3}^{* t}\right) \\
= & \lambda_{1} k_{1} k_{1}^{* t}+\left(\lambda_{2}-\lambda_{3}\right) k_{2} k_{2}^{* t} \\
& +2 \cdot \lambda_{3} \cdot \frac{1}{2}\left(k_{2} k_{2}^{* t}+k_{3} k_{3}^{* t}\right) .
\end{aligned}
$$

If $\alpha_{1} \leq \pi / 4$, it can be seen that, $\alpha_{s}=\alpha_{1}, k_{s}=k_{1}$, then the surface scattering model $T_{s}=k_{1} k_{1}^{* t}$, and the volume scattering model $T_{v_{-} 2}=(1 / 2)\left(k_{1} k_{1}^{* t}+k_{3} k_{3}^{* t}\right)$. In addition, $\alpha_{d}=\alpha_{2}, k_{d}=k_{2}$, and double-bounce scattering model $T_{d}=k_{2} k_{2}^{* t}$. From the fifth line of (28), we can solve surface scattering powers $m_{s}$ and double-bounce powers $m_{d}$ as

$$
\begin{aligned}
& m_{s}=\lambda_{1}-\lambda_{3}, \\
& m_{d}=\lambda_{2} .
\end{aligned}
$$

If $\alpha_{2} \leq \pi / 4$, then $\alpha_{s}=\alpha_{2}, k_{s}=k_{2}, T_{s}=k_{2} k_{2}^{* t}$, and $\alpha_{d}=\alpha_{1}, T_{d}=k_{1} k_{1}^{* t}$. The volume scattering model is $T_{v_{-} 2}=$ $(1 / 2)\left(k_{2} k_{2}^{* t}+k_{3} k_{3}^{* t}\right)$. From the last line of (28), we can solve surface scattering powers $m_{s}$ and double-bounce powers $m_{d}$ as

$$
\begin{aligned}
& m_{s}=\lambda_{2}-\lambda_{3}, \\
& m_{d}=\lambda_{1} .
\end{aligned}
$$

Because of $\lambda_{1} \geqslant \lambda_{2} \geqslant \lambda_{3} \geqslant 0$ and because the scattering powers are solved as (23), (25), and (26) or (27), (29), and (30), the scattering powers are all nonnegative values.

\section{Experimental Study}

To prove the efficacy of proposed Freeman/eigenvalue decomposition, the experiments are conducted on the Lband fully PolSAR data of San Francisco which were acquired by NASA/JPL ARISAR. The spatial resolution and range resolution are both about $10 \mathrm{~m}$ and the radar incidence angle is from $5^{\circ}$ to $60^{\circ}$. The used PolSAR data is open access from the internet [18]. The original image is shown in Figure 1, with the selected regions, which are used in the later tests. The size of the used PolSAR image in these experiments is $900 \times 1024$. Before expanding the coherency matrix, the Sigma filter is used to process the speckle of the PolSAR data.

In order to demonstrate the efficacy of the proposed hybrid Freeman/eigenvalue decomposition, the compared 


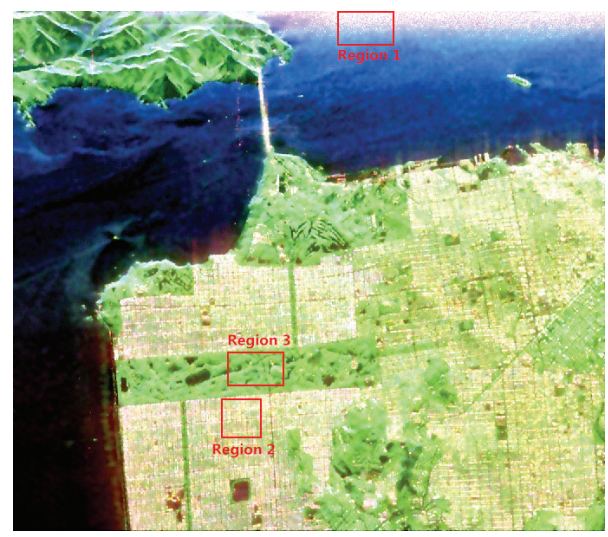

FIgURE 1: Original image of San Francisco.

TABLE 1: Means of dominated scattering powers in Region 1.

\begin{tabular}{lccc}
\hline & mean_ $m_{s}$ & mean $m_{d}$ & mean_ $m_{v}$ \\
\hline FDD 1 & 0.9192 & 0.0013 & 0.0794 \\
FDD 2 & 0.9475 & 0.0033 & 0.0492 \\
HFED 1 & 0.9348 & 0.0164 & 0.0488 \\
HFED 2 & 0.9348 & 0.0164 & 0.0488 \\
Proposed method & 0.9634 & 0.0053 & 0.0313 \\
\hline
\end{tabular}

methods used Freeman-Durden decomposition [8] (FDD 1), Freeman-Durden decomposition with the rotation of the coherency matrix [12] (FDD 2), hybrid Freeman/eigenvalue decomposition [14] (HFED 1), and hybrid Freeman/eigenvalue decomposition with extended volume scattering model [15] (HFED 2). The PolSAR data are decomposed into three components: surface scattering power $m_{s}$ (blue), doublebounce scattering power $m_{d}$ (red), and volume scattering power $m_{v}$ (green) in Figure 2 .

From Figures 2(a)-2(e), three main terrain types in the used data, that is, ocean areas, city blocks, and forests are reconstructed well by the scattering powers derived from these five decompositions. For further analysis, we compare the scattering powers in three selected regions in Figure 1. Those regions are Region 1, Region 2, and Region 3, respectively. The sizes of these regions are $60 \times 100,70 \times 70$, and $60 \times$ 100 in proper order. The types of ground truth are ocean areas, city blocks, and forests. The mean values of surface scattering power mean $m_{s}$, double-bounce scattering power mean $m_{d}$, and volume scattering power mean_m $m_{v}$ in the three regions are listed in Tables 1, 2, and 3. The mean scattering powers are all normalized by the total scattering powers (i.e., $m_{s}+m_{d}+$ $\left.m_{v}\right)$. In Region 1, it can be seen that mean $m_{s}$ given by the proposed decomposition is 0.9634 , which is about $4.8 \%, 1.7 \%$, $3.1 \%$, and $3.1 \%$ higher than the other four decompositions, respectively. The efficacy of all the five decompositions on Region 1 (sea) provides the excellent performance. In Region 2 , the average double-bounce scattering power mean $m_{d}$ of the proposed decomposition also outperforms the other methods. It is about $3.9 \%, 2.8 \%, 3.0 \%$, and $0.6 \%$ larger than these four decompositions in proper sequence. But, in Region 3 , mean $m_{v}$ given by HFED 1 is the best.
TABLE 2: Means of dominated scattering powers in Region 2.

\begin{tabular}{lccc}
\hline & mean_ $m_{s}$ & mean_ $m_{d}$ & mean_ $m_{v}$ \\
\hline FDD 1 & 0.1082 & 0.4307 & 0.4611 \\
FDD 2 & 0.1676 & 0.5017 & 0.3307 \\
HFED 1 & 0.1686 & 0.5008 & 0.3306 \\
HFED 2 & 0.3245 & 0.5137 & 0.1618 \\
Proposed method & 0.2404 & 0.5158 & 0.2438 \\
\hline
\end{tabular}

TABLE 3: Means of dominated scattering powers in Region 3.

\begin{tabular}{lccc}
\hline & mean_ $m_{s}$ & mean_ $m_{d}$ & mean_ $m_{v}$ \\
\hline FDD 1 & 0.0314 & 0.1535 & 0.8151 \\
FDD 2 & 0.0247 & 0.1555 & 0.8198 \\
HFED 1 & 0.0673 & 0.1098 & 0.9228 \\
HFED 2 & 0.0996 & 0.1097 & 0.7904 \\
Proposed method & 0.2380 & 0.0754 & 0.6865 \\
\hline
\end{tabular}

TABLE 4: Classification results (\%).

\begin{tabular}{lccc}
\hline & Region 1 & Region 2 & Region 3 \\
\hline FDD 1 & 100 & 36.49 & 95.97 \\
FDD 2 & 100 & 84.65 & 99.32 \\
HFED 1 & 100 & 84.82 & 99.47 \\
HFED 2 & 100 & 86.63 & 95.50 \\
Proposed method & 100 & 95.92 & 98.05 \\
\hline
\end{tabular}

We classified the selected regions into three classes. The rule is simple; that is, the max scattering power determines the label of the pixel. If the surface scattering power is the biggest in the three scattering powers, the pixel is labeled "surface scattering class." It can be seen that the true labels in Region 1, Region 2, and Region 3 are surface scattering class, double-bounce scattering class, and volume scattering class, respectively. The accuracy of the selected zones is listed in Table 4. It can be seen, in Region 1, that surface scattering powers of these decompositions are all the biggest ones, and, in Region 3, these classification accuracies are all higher than 95\%. In contrast to the other decompositions, the average scattering powers of the proposed method in Region 3 are not very good, but the classification accuracy is higher than $98 \%$. Moreover, in Region 2, the proposed method obtains the best results, as large as $95.92 \%$, which are about $59.43 \%, 11.72 \%$, $11.10 \%$, and $9.29 \%$ higher than FDD 1, FDD 2, HEFD 1, and HEFD 2, respectively.

\section{Conclusions}

In this paper, a novel version of hybrid Freeman/eigenvalue decomposition for polarimetric SAR data is proposed. Three conclusions can by drawn about the proposed method. Firstly, the eigenvectors of the rotated coherency matrix with reflection symmetry condition are used as surface scattering or double-bounce scattering models. Secondly, in contrast to conventional Freeman-Durden decompositions, the derived scattering powers are all nonnegative values. Thirdly, 


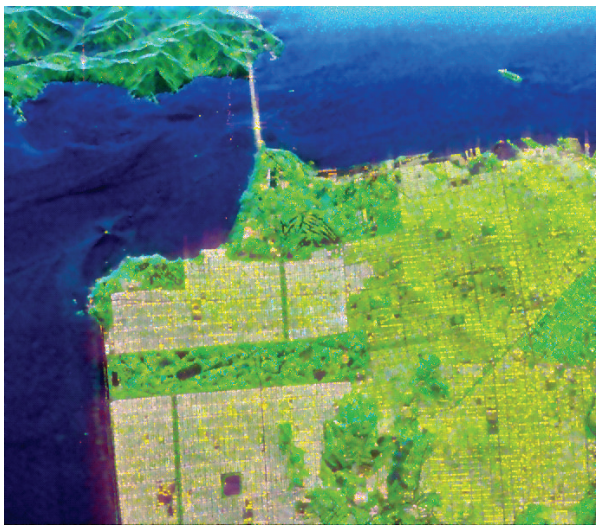

(a)

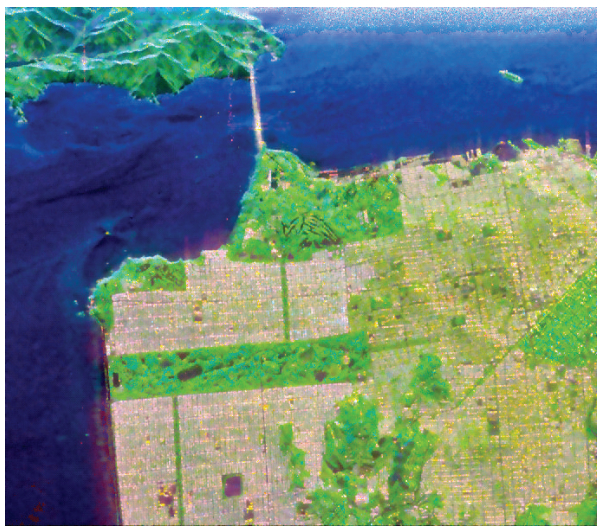

(c)

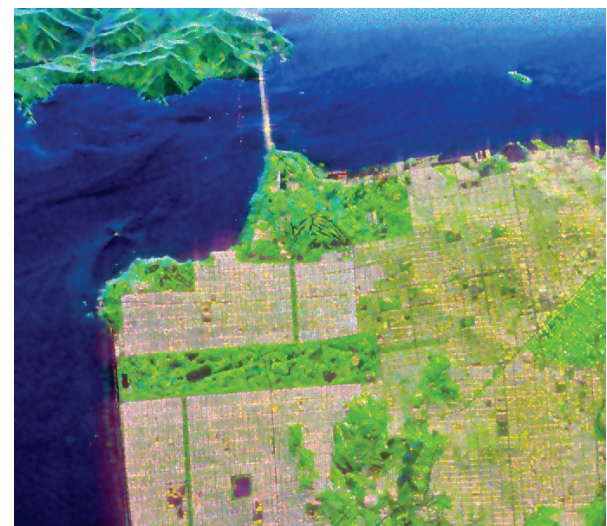

(b)

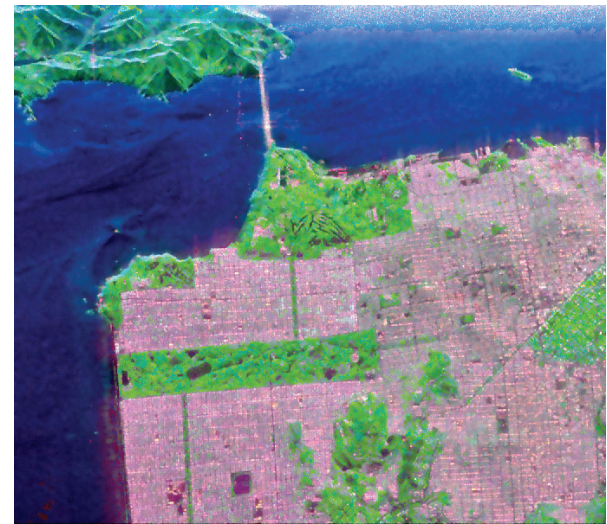

(d)

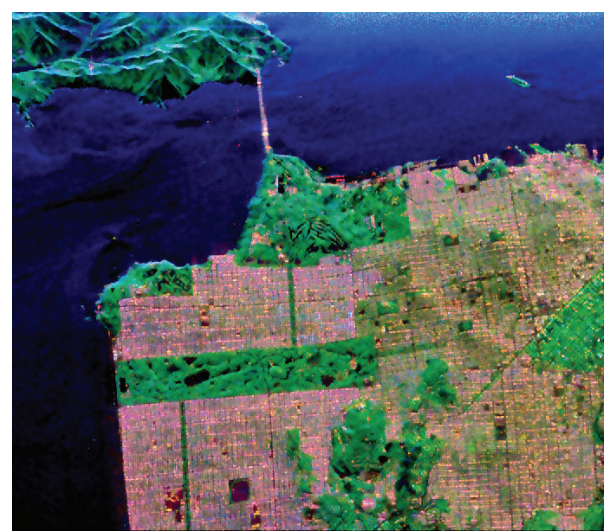

(e)

FIGURE 2: Decompositions of AIRSAR data on San Francisco with $m_{s}$ for blue, $m_{d}$ for red, $m_{v}$ for green. (a) FDD 1, (b) FDD 2 , (c) HFED 1 , (d) HFED 2, and (e) proposed decomposition.

the volume scattering model is determined by the entropy and anisotropy of the coherency matrix. Experimental results have proved the efficacy of the proposed decomposition.

\section{Conflict of Interests}

The authors declare that there is no conflict of interests regarding the publication of this paper.

\section{Acknowledgments}

This work was supported in part by the National High Technology Research and Development Program (863 Program) of China (no. 2013CB329402), the National Natural Science Foundation of China (nos. 61173092, 61377011, and 61272282), and the Fund for Foreign Scholars in University Research and Teaching Programs (no. B07048). 


\section{References}

[1] J. J. van Zyl, R. Carande, Y. Lou, T. Miller, and K. Wheeler, “The NASA/JPL three-frequency polarimetric AIRSAR system," in Proceedings of the International Geoscience and Remote Sensing Symposium (IGARSS '92), vol. 1, pp. 649-651, Houston, Tex, USA, May 1992.

[2] E. L. Christensen, N. Skou, J. Dall et al., "EMISAR: an absolutely calibrated polarimetric L- and C-band SAR," IEEE Transactions on Geoscience and Remote Sensing, vol. 36, no. 6, pp. 1852-1865, 1998.

[3] R. Horn, "The DLR airborne SAR project E-SAR," in Proceedings of the International Geoscience and Remote Sensing Symposium "Remote Sensing for a Sustainable Future" (IGARSS '96), vol. 3, pp. 1624-1628, Lincoln, Neb, USA, May 1996.

[4] S. Buckreuss, R. Werninghaus, and W. Pitz, "The German satellite mission TerraSAR-X," in Proceedings of the IEEE Radar Conference (RADAR '08), pp. 1-5, Rome, Italy, May 2008.

[5] http://www.asc-csa.gc.ca/eng/satellites/radarsat2.

[6] S. R. Cloude and E. Pettier, "A review of target decomposition theorems in radar polarimetry," IEEE Transactions on Geoscience and Remote Sensing, vol. 34, no. 2, pp. 498-518, 1996.

[7] S. R. Cloude and E. Pottier, "An entropy based classification scheme for land applications of polarimetric SAR," IEEE Transactions on Geoscience and Remote Sensing, vol. 35, no. 1, pp. 6878, 1997.

[8] A. Freeman and S. L. Durden, "A three-component scattering model for polarimetric SAR data," IEEE Transactions on Geoscience and Remote Sensing, vol. 36, no. 3, pp. 963-973, 1998.

[9] Y. Yamaguchi, T. Moriyama, M. Ishido, and H. Yamada, "Four-component scattering model for polarimetric SAR image decomposition," IEEE Transactions on Geoscience and Remote Sensing, vol. 43, no. 8, pp. 1699-1706, 2005.

[10] Y. Yamaguchi, A. Sato, W.-M. Boerner, R. Sato, and H. Yamada, "Four-component scattering power decomposition with rotation of coherency matrix," IEEE Transactions on Geoscience and Remote Sensing, vol. 49, no. 6, pp. 2251-2258, 2011.

[11] O. Antropov, Y. Rauste, and T. Hame, "Volume scattering modeling in PolSAR decompositions: study of ALOS PALSAR data over boreal forest," IEEE Transactions on Geoscience and Remote Sensing, vol. 49, no. 10, pp. 3838-3848, 2011.

[12] W. An, Y. Cui, and J. Yang, "Three-component model-based decomposition for polarimetric sar data," IEEE Transactions on Geoscience and Remote Sensing, vol. 48, no. 6, pp. 2732-2739, 2010.

[13] G. Singh, Y. Yamaguchi, and S.-E. Park, "General four-component scattering power decomposition with unitary transformation of coherency matrix," IEEE Transactions on Geoscience and Remote Sensing, vol. 51, no. 5, pp. 3014-3022, 2013.

[14] S. R. Cloude, Polarisation: Applications in Remote Sensing, Oxford University Press, London, UK, 2009.

[15] G. Singh, Y. Yamaguchi, S.-E. Park, Y. Cui, and H. Kobayashi, "Hybrid freeman/eigenvalue decomposition method with extended volume scattering model," IEEE Geoscience and Remote Sensing Letters, vol. 10, no. 1, pp. 81-85, 2013.

[16] F. Xu and Y.-Q. Jin, "Deorientation theory of polarimetric scattering targets and application to terrain surface classification," IEEE Transactions on Geoscience and Remote Sensing, vol. 43, no. 10, pp. 2351-2364, 2005.

[17] J. S. Lee and E. Pottier, Polarimetric Radar Imaging: From Basics to Applications, CRC Press, Boca Raton, Fla, USA, 2009.

[18] https://earth.esa.int/web/polsarpro/datasets.html. 

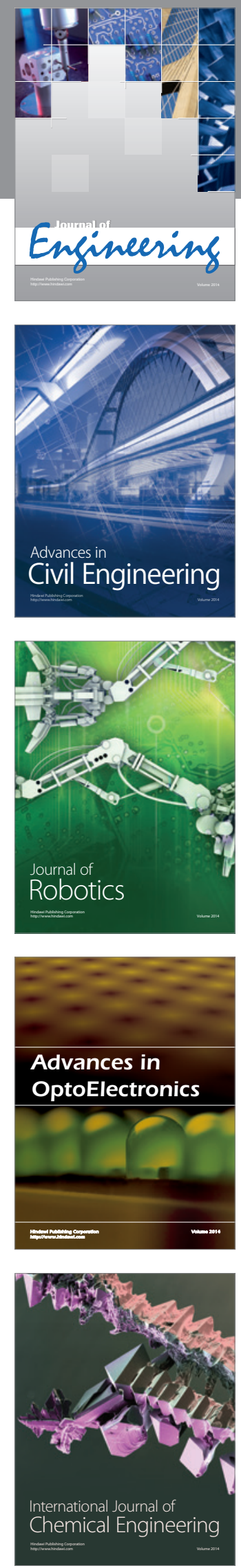

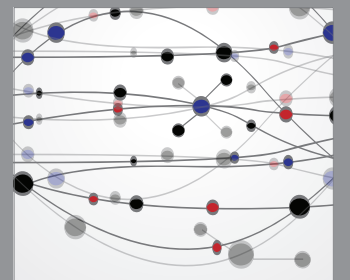

The Scientific World Journal
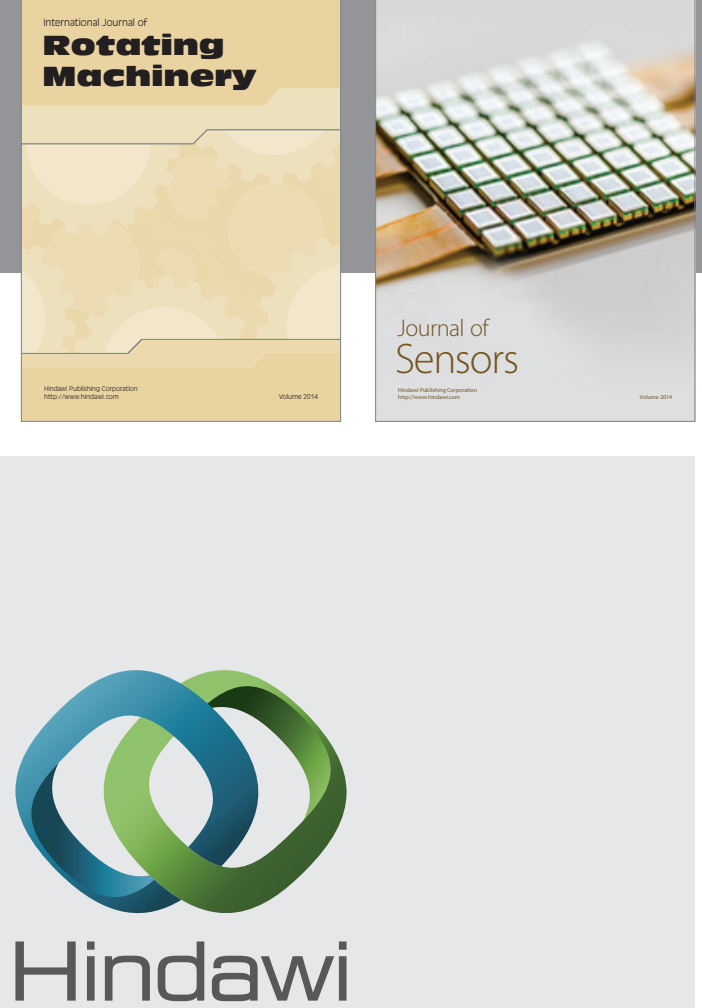

Submit your manuscripts at http://www.hindawi.com
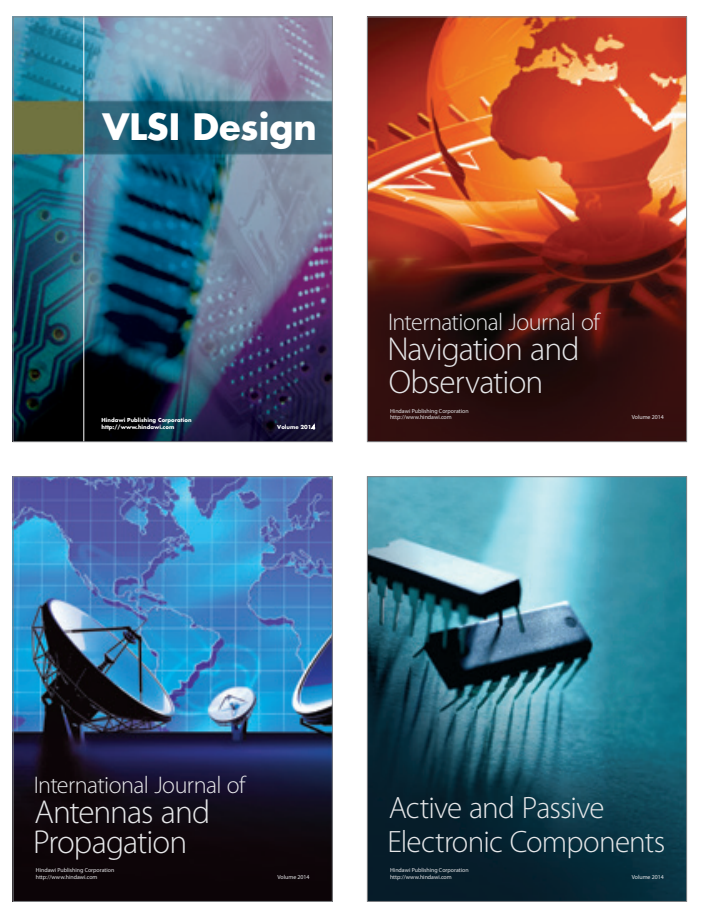
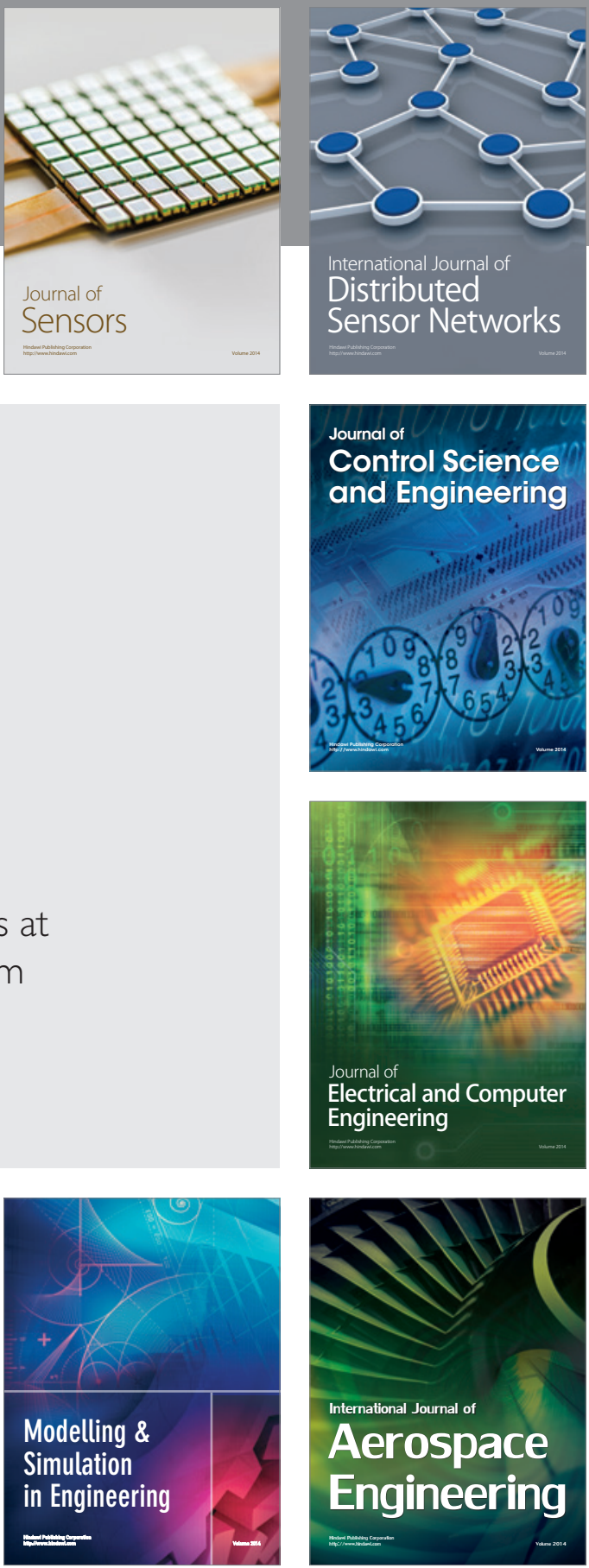

Journal of

Control Science

and Engineering
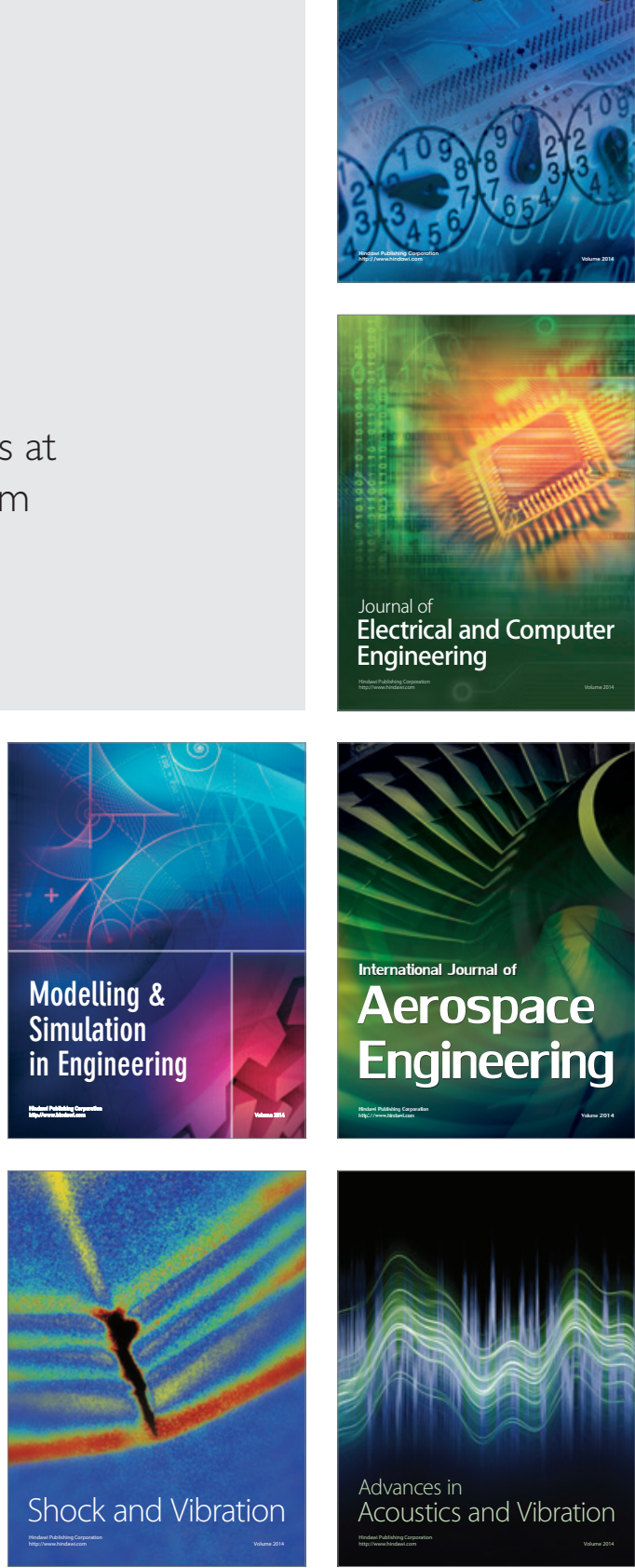